\title{
Das Opfer nach der Sintflut für die Gottheit(en) des Alten Testaments und des Alten Orients: Eine neue Deutung
}

\begin{tabular}{|c|c|}
\hline $\begin{array}{l}\text { Author: } \\
\text { Gerlinde Baum }\end{array}$ & ann $n^{1,2}$ \\
\hline $\begin{array}{l}\text { Affiliations: } \\
{ }^{1} \text { Altes Testame } \\
\text { Evangelische T } \\
\text { Philipps-Unive } \\
\text { Germany }\end{array}$ & $\begin{array}{l}\text { ent, } \\
\text { heologie, } \\
\text { rsität Marburg, }\end{array}$ \\
\hline $\begin{array}{l}{ }^{2} \text { Department } \\
\text { University of } \mathrm{P} \\
\text { South Africa }\end{array}$ & $\begin{array}{l}\text { Dld Testament, } \\
\text { retoria, }\end{array}$ \\
\hline $\begin{array}{l}\text { Corresponden } \\
\text { Gerlinde Baun }\end{array}$ & $\begin{array}{l}\text { ce to: } \\
\text { hann }\end{array}$ \\
\hline $\begin{array}{l}\text { Email: } \\
\text { baumann@sta } \\
\text { marburg.de }\end{array}$ & aff.uni- \\
\hline $\begin{array}{l}\text { Postal address } \\
\text { Fachgebiet Alt } \\
\text { Fachbereich E } \\
\text { Theologie, Phi } \\
\text { Universität Ma } \\
\text { Marburg, Gerr }\end{array}$ & $\begin{array}{l}\text { es Testament, } \\
\text { vangelische } \\
\text { lipps- } \\
\text { arburg, } 30532 \\
\text { nany }\end{array}$ \\
\hline $\begin{array}{l}\text { Dates: } \\
\text { Received: } 18 \mathrm{~J} \\
\text { Accepted: } 19 \mathrm{~J} \\
\text { Published: } 05\end{array}$ & $\begin{array}{l}\text { une } 2013 \\
\text { une } 2013 \\
\text { Sept. } 2013\end{array}$ \\
\hline $\begin{array}{l}\text { How to cite th } \\
\text { Baumann, G., } \\
\text { Opfer nach de } \\
\text { die Gottheit(e } \\
\text { Testaments un } \\
\text { Orients: Eine r } \\
\text { Verbum et Ecc } \\
\text { Art. \#888, } 7 \text { pa } \\
\text { http://dx.doi.c } \\
\text { ve.v34i2.888 }\end{array}$ & $\begin{array}{l}\text { is article: } \\
\text { 2013, 'Das } \\
\text { r Sintflut für } \\
\text { n) des Alten } \\
\text { d des Alten } \\
\text { leue Deutung', } \\
\text { lesia 34(2), } \\
\text { ages. } \\
\text { rg/10.4102/ }\end{array}$ \\
\hline $\begin{array}{l}\text { Note: } \\
\text { This article is } \\
\text { Jurie le Roux, } \\
\text { for continually } \\
\text { encounters! }\end{array}$ & $\begin{array}{l}\text { ledicated to } \\
\text { with thanks } \\
\text { inspiring }\end{array}$ \\
\hline $\begin{array}{l}\text { Copyright: } \\
\text { C 2013. The A } \\
\text { Licensee: AOS } \\
\text { OpenJournals. } \\
\text { is licensed unc } \\
\text { Creative Comn } \\
\text { Attribution Lic }\end{array}$ & $\begin{array}{l}\text { IS } \\
\text { Ihors. } \\
\text { This work } \\
\text { ler the } \\
\text { nons } \\
\text { ense. }\end{array}$ \\
\hline Read online: & \\
\hline 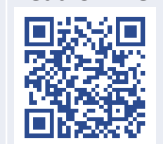 & $\begin{array}{l}\text { Scan this QR } \\
\text { code with your } \\
\text { smart phone or } \\
\text { mobile device } \\
\text { to read online. }\end{array}$ \\
\hline
\end{tabular}

The Sacrifice for (the) God(s) after the Flood in Ancient Israel and the Ancient Near East: A New Interpretation. The experience of a large, devastating flood is part of the cultural heritage of mankind. The famous 'texts of the deluge' come from Mesopotamia. Here, the flood tradition dates back to the 3rd Millennium. The longest and most traditional of these texts, which - amongst other things - deal with the interpretation of these events, is the Atramhasis myth. The literary-dependent text is the Gilgamesh epic, and the Old Testament version is the story of the Flood that is found in Genesis 6-9. For a long time the similarities and differences between these three texts have been known. However, so far little attention was given to a passage that all three texts share: the sacrifice of the surviving humans after the Flood. The reaction of the deity(ies) differs in these three texts. In this article I would like to consider the similarities and differences between the texts in order to evaluate the significance of the Old Testament text. This is against the background of recent insights in the field of ancient Israelite sacrifice, related to cultural anthropology. These three passages are first considered in their context and then compared to the relevant aspects of each other before a conclusion is drawn.

\section{Nach der Sintflut}

Die Erfahrung einer großen, vernichtenden Flut gehört zum kulturellen Erbe der Menschheit. ${ }^{1}$ Die bekanntesten 'Sintflutdichtungen' stammen aus dem Zweistromland, aber es gibt daneben auch Texte aus dem griechischen Raum, aus dem Gebiet des heutigen Libanon, aus Israel, Ägypten und Indien. In Mesopotamien reicht die Sintfluttradition bis ins 3. Jahrtausend zurück. Im westlichen Mittelmeer und im Vorderen Orient besitzt sie eine Nachgeschichte, die über die biblische Zeit hinausreicht. Die längsten, geschlossensten und am besten überlieferten Texte, die sich - unter anderem - mit der Deutung dieser Ereignisse befassen, sind der AtramhasisMythos sowie, literarisch davon abhängig, das Gilgameš-Epos. Im Alten Testament ist dies die sogenannte Sintfluterzählung Genesis 6-9.

Seit langem sind die Gemeinsamkeiten und Unterschiede zwischen diesen drei Texten bekannt. Wenig Beachtung fand dabei allerdings bisher eine Passage, die alle drei Texte gemeinsam haben: die Opferhandlung der überlebenden Menschen nach dem Ende der Sintflut. Die Reaktion der Gottheit(en) auf das Opfer fällt in den drei Texten unterschiedlich aus. In diesem Beitrag möchte ich die Gemeinsamkeiten und Unterschiede zwischen den Texten betrachten und in Hinblick auf die Bedeutung für das Alte Testament auswerten. Dies geschieht vor dem Hintergrund der neueren Erkenntnisse im Bereich des altisraelitischen Opfers. ${ }^{2}$ Dazu werden die drei Textpassagen zunächst in ihrem jeweiligen Kontext betrachtet ('Das Opfer nach der Sintflut in seinem jeweiligen Kontext') und dann in den relevanten Aspekten miteinander verglichen ('Die menschlichen Opfer und ihre Wirkung auf die Gottheit[en]'), bevor ein Fazit gezogen wird.

\section{Das Opfer nach der Sintflut in seinem jeweiligen Kontext Atramhasis $^{3}$}

Die am besten erhaltene altbabylonische Fassung des Atramhasis-Mythos stammt wohl aus dem 17. Jahrhundert vor Christus, aber es existieren auch teils spätere umfangreiche Neubearbeitungen. ${ }^{4}$ Der Mythos verknüpft die beiden Themen Menschenschöpfung und Flut miteinander, und die Textpassage über das Ende der Sintflut und das Opfer kommt am Ende des Mythos zu stehen. Die Menschen werden von den höheren Göttern (Anunnaku) erschaffen,

1.Einen Überblick über antike Sintflutsagen bieten zum Beispiel Caduff (1986), Schmidt (1995) oder García Martínez und Luttikhuizen (1998).

2.Einen sehr guten Überblick hierüber bietet Heft 1 des Jahrganges 56 (2011) von Verkündigung und Forschung; darin insbesondere die Beiträge von Willi-Plein (2011) und Eberhart (2011)

3.Siehe Lambert und Millard (1969); für die deutsche Übersetzung siehe von Soden (1994).

4.Von Soden (1994:612f.). Hier wird die immer noch einzige vollständige wissenschaftliche Ausgabe von Lambert und Millard (1969) zugrundegelegt. 
um den unteren Göttern (Igigi) die Arbeit abzunehmen und die Versorgung der Götter sicherzustellen. Später vermehren sich die Menschen allerdings unkontrolliert und machen viel Lärm bei ihrem Tun. Sie stören die Götter, und so fassen diese den Beschluss, die Menschen zuerst zu dezimieren und, als sich das nicht als wirksam erweiset, sie gänzlich auszurotten. Aufgrund der gezielten Indiskretion des 'weisen' Gottes Enki kann allerdings der 'überaus Weise' Atramhasis die Flut überleben. Während der Flut haben die Götter allerdings die Erfahrung gemacht, dass die Ausrottung der Menschen keine gute Idee war, denn sie litten Hunger und Durst, weil niemand sie mehr versorgte. Entsprechend ausgehungert und begierig nach dem Räucheropfer ${ }^{5}$ sind die Götter nach der Sintflut:

Atramhasis III v, 34f

34 [i-și-nu i-l] u e-re-ša

35 [ki-ma zu-ub-b] i e-lu ni-qí-i pa-ah-ru

\section{Übersetzung:}

34 [Die Göt]ter [rochen] den Duft,

35 sammelten sich [wie Fliegen] über dem Opfer.

Nach dieser Szene beschließen die Götter, dass es keine derartige Vernichtung der Menschheit mehr geben soll. An zwei Punkten verändern sie ihren Umgang mit den Menschen: Zum einen sollen von nun an nur noch die schuldigen Menschen bestraft werden und nicht mehr alle wie bislang, und zum anderen soll die Zahl der Menschen durch Kindersterblichkeit und Kinderlosigkeit bestimmter Gruppen klein gehalten werden.

Die Textszene mit dem Opfer steht zwischen dem Ende der Flut und der Abkehr der Götter von ihrer Strategie der Vernichtung der Menschen. Die Erzähllogik legt nahe, dass die Götter durch Atramhasis' Opfer aus einer echten Notlage befreit werden, weil sie endlich wieder 'zu riechen' bekommen.

Welche Bedeutung hat diese Szene etwa ein Jahrtausend später im Gilgameš-Epos?

\section{Gilgameš}

Die Gilgameš-Tradition geht auf das 3. Jahrtausend vor Christus zurück. Die 'kanonische', am besten und geschlossensten erhaltene Fassung des Epos datiert wohl ins 11. Jahrhundert vor Christus; wichtige Textfunde stammen allerdings aus der Bibliothek Assurbanipals in Ninive (7. Jahrhundert). ${ }^{8}$ Der uns vorliegende Text hat also bereits

5.Die Passage über die Opfermaterie ist leider nicht erhalten, aber es ist von einem Räucheropfer auszugehen; in Atramhasis III v., 39-40 ist es ein Räucheropfer: qúut-ri-ni (qutrinna, auch qataru I: Räucherwerk, Rauchopfer; bei Roth [1982:166] 'incence offering'); bei von Soden (1965:930): 'Weihrauch-(opfer)'.

6.Text nach: Lambert und Millard (1969:98).

7.Siehe siehe Maul (2005) für die deutsche Übersetzung des Textes.

8.Zur Textüberlieferung siehe Sallaberger (2008:9, 59-82). Der vorliegenden Darstellung liegt die wissenschaftliche Textausgabe von George (2003) zugrunde, in der alle bekannten Fragmente berücksichtigt sind. eine lange Vorgeschichte. Ursprünglich gehörte die SintflutEpisode wohl nicht zum Gilgameš-Stoff.

Wie ist die Opferszene ins Gilgameš-Epos eingebettet? Gilgameš ist ein Gott-Mensch und der Legende nach der mächtige König von Uruk in längst vergangener Zeit. Um seine Macht $\mathrm{zu}$ begrenzen, wird ihm ein Begleiter erschaffen, Enkidu. Beide werden enge Freunde und ziehen aus, um Abenteuer zu erleben. Allerdings stirbt Enkidu bald. Gilgameš ist untröstlich und macht sich auf die Suche nach dem ewigen Leben. Insbesondere sucht er nach dem einzigen Menschen, der das ewige Leben besitzt: Utnapišti [ich fand das Leben]. Nach langer und schwieriger Reise trifft Gilgameš auf Utnapišti. Dieser verrät ihm, wie er das ewige Leben erlangte - nämlich nach der Flut, die er als einziger Mensch mit seiner Familie überlebt hat. Damit hat Utnapišti im Gilgameš-Epos die gleiche Rolle inne wie Atramhasis im gleichnamigen Mythos. ${ }^{9}$ Die Fluterzählungen des GilgamešEpos und des Atramhasis-Mythos gehen dabei in Teilen parallel, teilweise stimmen sie sogar wörtlich überein. ${ }^{10}$ Auch die Opferszenen und die Reaktionen der Götter ähneln sich sehr:

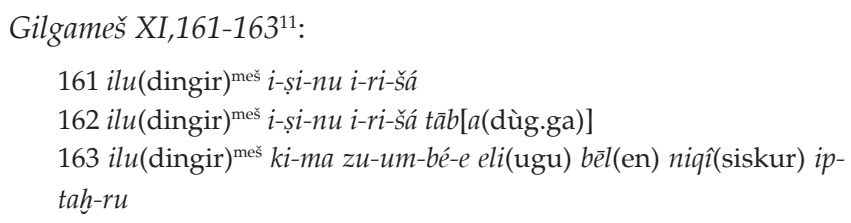

\section{Übersetzung:}

161 Die Götter rochen den Duft,

162 die Götter rochen den angenehmen Duft,

163 die Götter versammelten sich wie Fliegen um den Opfernden.

Auch hier wird den Göttern nach der Sintflut klar, dass die Vernichtung der Menschen ein Fehler war. Im Einzelnen verläuft das folgende Gespräch etwas anders als bei Atramhasis; so sind es im Detail andere Maßnahmen, die zukünftig für die kontrollierte Dezimierung der Menschen sorgen sollen. ${ }^{12}$ Gilgameš erfährt den Grund, aus dem Utnapišti von den Göttern das ewige Leben erhielt, und kehrt zurück nach Uruk. ${ }^{13}$

Die Erzählung der Erschaffung der Menschen, die im Atramhasis-Mythos der Sintflut vorausgeht, fehlt bei Gilgameš; statt dessen wird hier die Heldengeschichte von Gilgameš und Enkidu erzählt. Der Schwerpunkt der Fluterzählung liegt bei Gilgameš auf der Figur des Utnapišti und der Frage, wie dieser das ewige Leben erlangte. Die Sintfluterzählung ist hier also ein mythisches Element, das in den Rahmen des Epos eingebettet ist.

9.Utnapišti wird sogar an einer Stelle (Gilg XI 197) 'Atrambुasis' genannt; so Sallaberger (2008:76).

10.Sallaberger (2008:76)

11.George (2003:712)

12.Nun sind es wilde Tiere, Hunger und die Pest (genauer: Löwe, Wolf, Hunger und Erra).

13.Maul (2005:40): 'Endlich kann der müde, aber nun zu Einsicht und Weisheit gelangte Held, der die ganze Welt durchmessen hat, sich mit aller Erfahrung und Kraft seiner Aufgabe als "guter Hirte" widmen. Erst hierdurch wird sich Gilgamesch ewigen Ruhm erwerben.' 
In der Gilgameš-Fluterzählung werden die Götter dreimal an den Anfang einer Zeile gesetzt, wodurch die Bedeutung gerade dieser Zeilen stärker hervorgehoben wird. Der gleiche Effekt wird, wie George (2003) beobachtet, auch durch die jeweils länger werdenden Textzeilen erreicht. ${ }^{14}$ Im Unterschied zu Atramhasis erhält hier der Opferduft das Attribut tābum, 'süß, angenehm'. Auch sammeln sich die Götter nicht um das Opfer, sondern um den Opfernden; wiederum ein Zeichen dafür, dass die Person des Utnapišti von größerer Bedeutung ist als Atramhasis im gleichnamigen Mythos. ${ }^{15}$

Darin wird deutlich, dass auch bei der stellenweise fast wörtlichen Übernahme von Teilen aus Atramhasis bei Gilgameš die altorientalische Tradition jeweils neue und eigene Akzente setzt. Der ältere Text wird eben nicht wortgleich wiedergegeben, sondern er erhält eine eigene, an den jeweiligen Kontext angepasste Schwerpunktsetzung.

\section{Genesis 8:20f.}

Während die Verse Genesis 8:20f. häufig ausgelegt worden sind, ${ }^{16}$ trifft das auf das Opfer Noahs für Gott nicht zu: Ihm wurde bisher nicht so viel Aufmerksamkeit zuteil wie den sich anschließenden Worten JHWHs.

Die Verse Genesis 8:20f. sind eingebunden in den größeren literarischen Zusammenhang der biblischen Fluterzählung Genesis 6-9, die wiederum einen Teil der biblischen Urgeschichte (Gen 1-11) bildet. Wie beim AtramhasisMythos verklammert die biblische Urgeschichte die Erzählungen von Menschenschöpfung und Flut. Allerdings finden wir in der Genesis zwei unterschiedliche Versionen der Menschenschöpfung (P: Gen 1:1-2, 4a; 'J': Gen 2:4b-3:24) mit jeweils unterschiedlichen Schwerpunktsetzungen, die in der Fluterzählung eine gemeinsame Fortsetzung finden. Auch besteht die Urgeschichte der Genesis noch aus weiteren Elementen - dem ersten Mord der Menschheitsgeschichte (Gen 4, Kain und Abel), zwei Genealogien (Gen 5 und Gen 10) sowie der Erzählung vom Turmbau in Babylon (Gen 11).

Die Urgeschichte in ihrer jetzigen Form geht höchstwahrscheinlich auf die nachexilische Zeit Israels zurück. Ältere Teile wie die 'jahwistischen' sind dabei möglicherweise in die Königszeit $\mathrm{zu}$ datieren. In der biblischen Fluterzählung fasst JHWH zunächst den Beschluss, die Lebenszeit der Menschen zu begrenzen (Gen 6:3). Dann aber bereut er auch die Menschenschöpfung als solche, weil 'alles Dichten und Trachten ihres Herzens nur böse war $^{\prime 17}$ (Gen 6:5). Also sollen die Menschen und alle

14.'These three lines grow steadily longer in length and complexity, a literary device
that neatly captures the intensifying accumulation of gods at the gathering'
(George 2003:518). Bibel', so Seebass (1996:220).

17.Übersetzung der Lutherbibel (1984). anderen Lebewesen ausgerottet werden. Allein Noah bleibt bewahrt, weil er gerecht ist $(\mathrm{P})$, beziehungsweise Gefallen gefunden hat vor JHWH ('J'). Er soll sich die Arche bauen, einen großen schwimmbaren Kasten, in dem auch seine Familie sowie zwei (P; 7:8f.) beziehungsweise sieben ('J'; 7:2f.) Tierpaare Platz finden. Die Flut setzt ein und vernichtet alles Leben auf Erden. Nach dem Ende der Flut ruft JHWH schließlich Noah samt Familie und Tieren aus der Arche heraus. Dann folgt die Opferpassage:

Genesis 8:20f.:

20

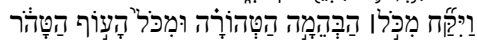

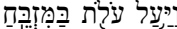
21

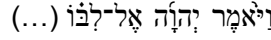

Übersetzung:

20 Noah baute einen Altar für JHWH;

er nahm von allem kultisch reinen Vieh und von allen reinen Vögeln

und ließ ein Brandopfer von dem Altar aufsteigen.

21a JHWH roch den 'beruhigenden' Geruch,

und JHWH sprach zu sich: (...)

Nach dem 'Riechen' nimmt JHWH sich vor, die Erde nicht noch einmal $\mathrm{zu}$ vernichten wegen der Menschen, auch wenn 'das Dichten und Trachten des menschlichen Herzens ... böse ist von Jugend auf' (Gen 8:21) (Übersetzung der Lutherbibel [1984]). Für die Bestandszeit der Erde sollen sich die Rhythmen der Natur abwechseln, ohne dass sich die Zerstörung durch Gott wiederholt.

Die Verse Genesis 8:20-22 bilden innerhalb der Flutgeschichte nach Ansicht der Exegese einen ersten 'Beschluss' 18 beziehungsweise den 'Epilog der "jahwistischen" Flutgeschichte' ${ }^{19}$ Die Verse beenden allerdings nicht die biblische Fluterzählung insgesamt. Diesen Abschluss stellen die - priesterschriftlichen - Verse Genesis 9:1-17 mit Segen und Bundesschluss dar. Unser Text beschließt also erst einmal nur einen Erzählstrang der biblischen Flutgeschichte, nicht aber die gesamte Erzählung. Deshalb bildet er nur eingeschränkt eine Parallele zu den Sintfluterzählungen der altorientalischen Texte, weil dort durch die Rücknahme des Vernichtungsbeschlusses jeweils der gesamte Abschnitt beendet wird.

Damit haben wir uns einen Überblick über die drei Texte mit ihren Sintflut- und Opfererzählungen verschafft. Wie ist nun jeweils im Einzelnen das Opfer und die göttliche Reaktion darauf $\mathrm{zu}$ bewerten?

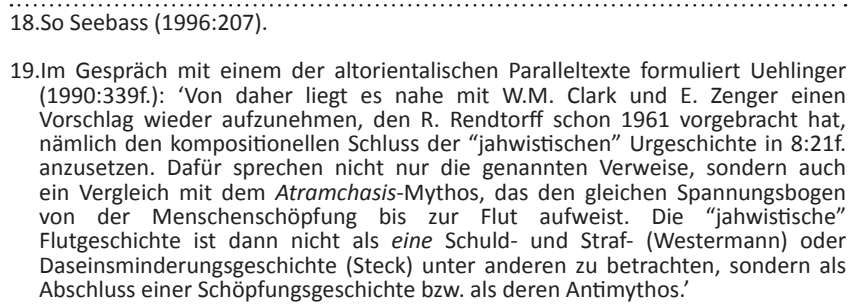

19.Im Gespräch mit einem der altorientalischen Paralleltexte formuliert Uehlinger (1990:339f.): 'Von daher liegt es nahe mit W.M. Clark und E. Zenger einen Vorschlag wieder aufzunehmen, den R. Rendtorff schon 1961 vorgebracht hat nämlich den kompositionellen Schluss der "jahwistischen" Urgeschichte in 8:21f anzusetzen. Dafür sprechen nicht nur die genannten Verweise, sondern auch ein Vergleich mit dem Atramchasis-Mythos, das den gleichen Spannungsbogen von der Menschenschöpfung bis zur Flut aufweist. Die "jahwistische" Flutgeschichte ist dann nicht als eine Schuld- und Straf- (Westermann) oder Daseinsminderungsgeschichte (Steck) unter anderen zu betrachten, sondern als Abschluss einer Schöpfungsgeschichte bzw. als deren Antimythos.' 


\section{Die menschlichen Opfer und ihre Wirkung auf die Gottheit(en) \\ Atrambuasis und Gilgameš}

Die Wirkung des Opfers nach der Flut ist sowohl im Atramhasis-Mythos als auch im Gilgameš-Epos durch ein gemeinsames Merkmal gekennzeichnet: Die Götter 'riechen' den (bei Gilgameš 'angenehmen') 'Duft' des Opfers und sammeln sich 'wie Fliegen' um das Opfer (Atr) beziehungsweise den Opferer (Gilg).

Der Vergleich mit den Fliegen ist in der altorientalistischen Forschung häufig als despektierliche Darstellung eingeordnet worden. ${ }^{20}$ Anne Kilmer (1987) allerdings hat sich dieser Symbolik stärker gewidmet und eine interessante Entdeckung gemacht, die dazu Anlass gibt, dieses Urteil zu revidieren: Das Fliegenmotiv kommt sowohl vor als auch nach dem Bild für die vermeintlich 'gierigen' Götter vor. Bereits in Atramhasis III iii 19 ist davon die Rede, dass während der Flut ein Flattern 'wie Fliegen' (ki-ma zu$u b-b i) \mathrm{zu}$ beobachten ist. In Atramhasis III 44f. spricht die Muttergöttin davon, dass die Geschöpfe 'ohne (Hilfe von) mir ... wie Fliegen' wurden. ${ }^{21}$ In Atramhasis IV 6f. füllen die in der Flut treibenden Leichname der Menschen den Fluss wie 'Schlammfliegen' (zoologisch: sialis lutaria; akkad. kulïlu). Unmittelbar nach der Opferszene legt die Muttergöttin ihr 'Fliegengeschmeide' an. Darunter ist eine aus Lapislazuli gearbeitete Kette aus Fliegennachbildungen zu verstehen, für die es auch archäologische Zeugnisse gibt. ${ }^{22}$ Diese Kette hat Anu der Muttergöttin geschenkt. Nun legt sie diese zur Erinnerung an den Tag der von Anu gebrachten Flut an, den sie nicht vergessen will (Atr III v 46f.; III vi 2; Gilg XI, 164f.), weil hier die von ihr geschaffenen Menschen vernichtet wurden. Kilmer wertet diese Erwähnungen der Fliegen so: 'What is clear is that the image of flies relates to death and dead things' (Kilmer 1987:177). Daneben sind Fliegen ikonographisch auch im Kriegskontext zu finden; sie finden sich über den in die Schlacht ziehenden Soldaten und in Ägypten als eine Art militärische Tapferkeitsmedaille nach bestandener Schlacht. ${ }^{23}$ Im Anschluss an Kilmer ist also das Bild der Gottheiten, die wie Fliegen um das Opfer beziehungsweise den Opferer herumfliegen, wohl nicht so sehr ein despektierliches. ${ }^{24}$ Hier wird Bezug auf den voraufgehenden und auf den folgenden Text genommen, in dem es vor allem um das Sterben der Menschen geht. Mit Claus Wilcke lässt sich dies so deuten:

Der Vergleich spiegelt das Schicksal der Menschen in dem der vor Hunger und Durst (fast, müssen wir sagen) toten Götter. Die Sintflut hat Götter wie Menschen fliegengleich gemacht - eine

20.So etwa bei George (2003:518): 'The simile used to describe the god's arrival is famously the image of hungry flies buzzing around a piece of food. This imagery implies a somewhat cynical view of gods, even more disrespectful than the earlie simile likening them to cowering dogs (SB XI 116).

21.So die Übersetzung von Wilcke (1999:92 mit Anm. 56).

22.So Hecker (1994:734, Anmerkung 163a); ebenso Lambert und Millard (1969:163f.).

23.Kilmer (1987:178) mit Bezug auf Porada (1972:173-175): '.. the fly symbolizes escape from death, or a near brush with death as well.' Daneben hebt Kilmer anhand anderer Textstellen hervor, dass die Fliege überall hingelangt und dass ihre schillernden Flügel an den Regenbogen erinnern (178f.).

24.Wilcke (1999:94), zur Stelle (mit Bezug auf Kilmer): 'Das ist kein despektierliches Bild.'
Erinnerung an den ersten Vers des Epos: Als die Götter Mensch waren. (1999:94f.)

Über das Fliegen-Motiv wird also das Schicksal der Götter mit dem der Menschen eng verknüpft: Wenn die Götter die Menschen umbringen, dann sind sie selbst auch vom Tod bedroht. Deshalb soll es auch nach den mesopotamischen Texten keine vernichtende Flut mehr geben. Das Motiv der Fliegen ist demnach keine despektierliche Rede über altorientalische Gottheiten, sondern ein Hinweis auf die Verwobenheit von göttlichem und menschlichem Schicksal. Es wird in den beiden diskutierten mesopotamischen Texten in ähnlicher Weise verwendet.

\section{Genesis 8:20-21a}

Zwar kommt die vermeintlich 'despektierliche' Fliegenmotivik in Genesis 8 nicht vor. Doch bereits die Schilderung Gottes als 'riechend' ist in der christlichen Tradition negativ rezipiert worden: Für die von griechischen, meist neuplatonischen Paradigmen geprägte Auslegungstradition ist dies eine besonders anstößige 'anthropomorphe' Redeweise von Gott. ${ }^{25}$ Man darf sich Gott, der doch gerade kein Mensch ist, nicht als riechend vorstellen - und schon gar nicht in der Weise, dass sich Gott womöglich durch einen Duft in seinem Handeln beeinflussen ließe.

Doch genau davon ist in unserem Text die Rede, wie ich im Folgenden ausführen möchte. Dafür zunächst ein genauerer Blick auf das 'Riechen' (רוח [II] $]^{26}$ Hif.). Diese Handlung wird JHWH nicht nur an dieser Stelle im Alten Testament zugeschrieben, sondern noch an drei weiteren: Nach Levitikus 26:31 will JHWH den ריח ניחהכם ('eurer Opfer') nicht mehr riechen, wenn Israel nicht auf ihn hört;, ${ }^{27}$ ganz ähnlich Amos 5:21, wo JHWH die 'Versammlungen' nicht riechen mag. 1 Samuel 26:19 wird das 'Speiseopfer'28 (מנחה) als gezielte Strategie von Saul vorgeschlagen, falls JHWH David gegen ihn aufwiegeln würde; dann solle man JHWH den Geruch der מנחה riechen lassen und würde auf eine Veränderung

25.So zum Beispiel Benno Jacob bereits mit kritischer Spitze gegen die Auslegungstradition: 'Ungebührlich strenggenommen wird der Ausdruck וירו, als sei er ein krasser Anthropomorphismus. Dass ריח ניחח ein solcher sei, wird nicht behauptet, dann aber ist das allein angemessene Verbum der Wahrnehmung וירח, das nicht anthropomorpher ist als Sehen und Hören, und seine sinnliche Urbedeutung nur für uns stärker herauskehrt. Außerdem aber ist der Ausdruck hochemphatisch ... [mit Verweis auf Levitikus 26,31 und Amos 5,21], ein Ton, de in der Stimmung Gottes vor der Flut $(6,6 f$.) erklungen war und jetzt verstummen soll' (Jacob 1934:227). Ähnlich Westermann (1976:608): 'Der Anstoß an diesem "anthropomorphen" Satz ist sehr alt ... und hält sich bis in die Gegenwart durch.'

26.Umstritten ist, ob es eine oder zwei Wurzeln $(\sqrt{ })$ des Verbs רוח gibt: Köhler und Baumgartner (2004) postulieren eine Wurzel mit den Bedeutungen A (die nur im Qal und Pual belegt ist, mit der Bedeutung 'sich ausweiten, sich verbreiten'; das Hifil wäre 'sich erleichtert fühlen') und B (nur im Hifil belegt: 'riechen'): 'Somit dürfte es nahe liegen, mit nur einer $\sqrt{n}$ zu rechnen, ohne dass die Existenz von durfte es nahe liegen, mit nur einer $\sqrt{ }$ m $z$ zu rechnen, ohne dass die Existenz von
zwei verschiedenen $\sqrt{ }$ ausgeschlossen wäre. Wir müssen die Frage offen lassen zwei verschiedenen $\sqrt{ }$ ausgeschlossen wäre. Wir müssen die Frage offen lassen
und geben der Nähe der beiden, sich aber doch unterscheidenden $\sqrt{\text { dadurch }}$ Ausdruck, dass wir das eine unter A und das andere unter B behandeln' (Köhler \& Baumgartner 2004:1116). Kronholm (1993:383) nimmt dagegen nur dieses eine Verb an, wobei er allerdings auch die Existenz zweier Wurzeln nicht ausschließt: 'Doch zieht man in der neueren Forschung nicht selten die Annahme einer einzigen gemeinsemit. Wurzel $r w h / r j h$ vor und führt dabei akk. napašu "weit werden", aber auch "blasen, atmen" (...) als Parallele an.' Gesenius geht für "רוח, von zwei Wurzeln aus (Gesenius 1962:748 sowie Gesenius 2009:1224f.).

27.Dazu vor allem Rendtorff (2004:63-69).

28.Zur mincha vergleiche Willi-Plein (1993:84): 'Als Begleitopfer zu anderen Opfersorten, also als "Speiseopfer", das aus Mehl oder Grieß in Kuchenform zubereitet und verbrannt wird, erfordert die Mincha auch Wohlgerüche und ein Wein-Gußopfer. Beides ist in den Rezepten auf das jeweilige Opfertier abgestimmt. Wein-Gußopfer. Beides ist in den Rezepten auf das jeweilige Opfertier abgestimmt.
Im ausgebauten Ritual ist die Mincha das "Zubrot" zum zubereiteten Fleisch des Tieropfers.' 
seiner Haltung hoffen. Diesen Stellen könnte man eine kritische Haltung gegenüber dem göttlichen Riechen entnehmen, weil sie die Möglichkeit der Manipulation Gottes beinhaltet. Allerdings machen zwei andere Texte deutlich, dass die Fähigkeit zu riechen in der Sicht des Alten Testaments ein konstitutives Attribut einer Gottheit ist: In Deuteronomium 4:28 wird Israel angekündigt, dass es einst Gottheiten verehren wird, die 'weder sehen noch hören, weder essen noch riechen können'. Ganz ähnlich Psalm 115:6: Die 'falschen' Gottheiten 'haben Ohren und hören nicht, sie haben eine Nase und riechen nicht'. Im Alten Testament wird also gerade keine durchgängig negative Sicht auf das Riechen von Gottheiten vertreten, sondern dieses Attribut als konstitutiv für eine Gottheit angesehen.

Dabei ist nicht unwichtig, was JHWH riecht. Das Objekt ריח .ריח הניחה des göttlichen Riechens heißt im Hebräischen bezeichnet den 'Geruch', 'Duft', 'Hauch beziehungsweise Atem'. ${ }^{29}$ Was sich allerdings hinter ניחח verbirgt, ist nach dem Urteil der Auslegenden nicht so eindeutig. Relativ neutral sind noch Übersetzungen mit 'beruhigend'30 oder 'besänftigend's1, vielleicht mit einer leichten Steigerung der Bedeutungsintensität als 'beschwichtigend'. ${ }^{32}$ Dabei wird immer ein Zusammenhang mit dem göttlichen Zorn impliziert, der länger oder kürzer andauert, denn ein ruhiger Gott muss schwerlich beschwichtigt werden. In eine andere Bedeutungsrichtung gehen Übersetzungen, die ניחה als 'wohltuend' oder 'angenehm' deuten. ${ }^{33}$ Eine ähnliche Bedeutungsnuance stützen die antiken Übersetzungen:

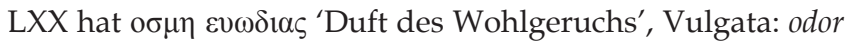
suavis, [Parfüm] oder odor suavitas, [Geruch der Lieblichkeit, Annehmlichkeit]. Auch die Übersetzung der Lutherbibel lautet 'mit lieblichem Geruch'.

An dieser Stelle scheint die Sekundärliteratur wegen der großen Unterschiede der Deutungen und der offensichtlichen Schwierigkeit, hier zu einem begründeten Urteil zu kommen,

29.Für die von Gesenius postulierte zweite Wurzel (Gesenius 1962:748 sowie Gesenius 2009:1224f.).

30.So zum Beispiel Eberhart (2002:369f.); Marx (2000:138): 'beruhigender Duft' Gesenius (2007:814): 'Beruhigung, Beschwichtigung'; stets in der Verbindung mit ריח: 'Beruhigungsduft, der angenehme Duft von Opfern für Jahwe zu dessen Beruhigung und Besänftigung'; desgleichen Schüle (2009:144).

31.Willi-Plein (2011:24): 'Alle Opfermaterie wird durch Verbrennung auf dem Altar an Gott übergeben, und zwar als "Besänftigungsgeruch für JHWH".

32.Von der 'Beschwichtigung' JHWHs durch den Opferduft spricht Koch (1986:443 mit Bezug auf Köhler [1966:177]) basierend auf Ezechiel 5:13 ('ich beschwichtige meinen Zor gegen sie'). All verwendet, 'dess ubertragen werdist, aber in völli (רוח Hifil mit (2004:1116] beziehungsweise ניחה als 'Beschwichtigung, mit רeschwichtigungsgeruch' [2004:657]). Ähnlich auch Stolz (1984:46) mit Verweis auf die verwandten altorientalischen Vorstellungen: 'Eine ganz spezifische, im Kult beheimatete Vorstellung komm in der Wortverbindung reach nichoach zum Ausdruck: Vom Opfer her steigt ein "Beschwichtigungsgeruch" zur Gottheit auf und bringt so das Verhältnis zwischen Mensch und Gott in Ordnung; die Altertümlichkeit der Vorstellung liegt auf de Hand (Speisung des Gottes durch den Geruch). Der Ausdruck kommt in Gen 8:21 (J) vor, ist aber im Zusammenhang mit dem Sintflutabschluss schon vorisraelitisch belegt (akk. erišu "Duft" in Gilg. XI, 159f.).' Bei Seebass dagegen divergieren die Bedeutungen von ריח ניחה: In der zusammenhängenden Textübersetzung (1996:203) riecht JHWH den 'lieblichen Duft', während ihm der Textkommentierung zufolge der 'Beschwichtigungsgeruch' (1996:221) in die Nase steigt.

33.So Koch (1986:445): 'Für P und Ez bedeutet reach nichoach ... der "wohltuende Geruch", der beim festlichen Opfer entsteht und das rechte Klima für den segnenden Umgang Gottes mit der Kultgemeinde schafft. Deshalb erwartet Ezechiel 20:41 davon "Wohlgefallen" (rāssāh ; vgl. 1 QS 3,11; 9,5; 1 QM 2,5; 11 QPsa 154)'; ähnlich Zwickel (1994:369), allerdings ohne weitere Erklärung: 'wohltuender Geruch'. nicht weiterzuführen. Wenden wir uns deshalb dem hebräischen Wort selbst zu.

Das Adjektiv ניחח ist - nach dem immer noch wegweisenden Artikel von Klaus Koch (1986:442) - ein erstarrtes Polel des Verbs נוח. Die Grundbedeutung von נוח Qal ist 'sich niederlassen, bleiben, wohnen, ruhen' (Gesenius 2007:792). So lässt sich etwa die Arche (Gen 8:4) auf dem Gebirge Ararat nieder oder der Geist JHWHs (Num 11:25f.) auf den 70 Ältesten Israels sowie (Jes 11:2) auf dem erwarteten Davidspross. Das Polel entspricht in der Bedeutung in etwa dem Piel und hat damit - Ernst Jenni (1981) zufolge gegenüber dem Qal 'faktitive und resultative Bedeutung' ${ }^{34}$ Diesen Überlegungen entsprechend ist ניחה also das, was ein 'Ruhen' bewirkt-oder aber ein 'Sich-Niederlassen'. Vielleicht könnte man die Bedeutung von ניחז im Deutschen am ehesten so umschreiben, dass dieser Opferduft 'einladend' ist, sich niederzulassen.

Jenseits der isolierten Bedeutung des Wortes ניחה fällt auf, dass die gesamte Wendung ריח (ה)ניחה im Alten Testament relativ häufig anzutreffen ist, und zwar im Kontext von Opferbestimmungen in Levitikus und Numeri sowie bei Ezechiel. Diese Texte sind allesamt zur priester(schrift)lichen Tradition $\mathrm{zu}$ rechnen. Bei keinem dieser Texte wird diskutiert, ob mit dem Opfer eventuell ein göttlicher Zorn 'besänftigt' oder ein aufgebrachter Gott 'beschwichtigt' werden muss. Dies spricht mit Blick auf Genesis 8:21 für die gerade vorgeschlagene Deutung, wonach der Opferduft ריח (ה)ניחה JHWH dazu bringt, zu ruhen oder sich niederzulassen.

Daneben hat das schwerpunktmäßige Vorkommen von ריח (ה)ניחה auch eine quellenkritische Implikation: Außer in Genesis 8:21 kommt die Wendung ausschließlich in P-Texten vor; Genesis 8:21 wäre der einzige Beleg für ein Vorkommen außerhalb von P. So legt sich die Vermutung nahe, dass die Wendung ריח הניחה in Genesis 8:21 eigentlich in anderen Textzusammenhängen beheimatet ist und von dort aus hierher transferiert wurde. Es scheint sowohl von der Textchronologie als auch von der Textentstehung her aus anderen Texten in diesen in der Textchronologie weit vorne stehenden und ursprünglich älteren Zusammenhang 'eingewandert' zu sein. In Genesis 8:20f. wird durch die Wendung ריח הניחה der Ursprung des Brandopfers zurückverlegt in die Urgeschichte. Damit unterscheidet sich Genesis 8:20f. von Genesis 4:3; dort ist nicht dezidiert vom Brandopfer die Rede, sondern von מנחה, und hier wird nicht vom Riechen JHWHs, sondern vom Sehen JHWHs gesprochen. Durch die Positionierung innerhalb des Sintflut-Zusammenhangs bekommt das Opfer in Genesis 8:20f. zudem eine universale Bedeutung: Es wird nicht nur in Israel gebracht, sondern Noah als Ur-Mensch der nachsintflutlichen Neuschöpfung und Nichtisraelit bringt es, und zwar für JHWH.

Erhellend ist darüber hinaus auch eine Deutung des Opfers in Genesis 8:20f., wenn neuere Opfertheorien herangezogen

34.Jenni (1981:155); dort weiter: "d.h. das Subjekt bewirkt am Objekt den von dem entsprechenden Adjektiv oder vom passiven Partizip des Grundstamms
ausgesagten Zustand Öfter wird der adjektivisch ausgedrückte Zustand nicht objektiv bewirkt, sondern nur subjektiv als vorhanden erklärt.' 
werden. ${ }^{35}$ Danach wird das Opfer zunehmend weniger mit seiner Fokussierung auf Sühne oder Vergebung verstanden, sondern stärker im Rahmen eines religionswissenschaftlichen Verständnisses als Gabe, durch welche eine Kommunikation mit Gott hergestellt oder fortgeführt werden soll. ${ }^{36}$ Als Vorreiter dieser neuen Opferdeutung können Alfred Marx und Christian Eberhart gelten. ${ }^{37}$ In dieser Sichtweise wird das Opfer vor dem Hintergrund der Gepflogenheiten am altorientalischen Königshof als Gastmahl für Gott als einen Höherstehenden verstanden. Das gilt in Israel insbesondere für das Brandopfer, wie Ina Willi-Plein bereits 1993 betont hat. ${ }^{38}$ Durch die Gabe des ganzen Tieres für Gott wird dieser im Brandopfer wie ein vornehmer Gast behandelt. Man lädt ihn ein und sucht seine Nähe, indem ihm nur das Beste vorgesetzt wird ${ }^{39}$ In diesem Verständnis des Opfers geht es in der Begegnung um Kontakt und Kommunikation und um den Austausch mit dem Ehrengast. ${ }^{40}$ Marx präzisiert dies noch einmal vor dem Hintergrund des 'Altargesetzes' in Exodus 20:22-26, der Ätiologie des Opfers im Alten Testament: 'Das Opfer dient nicht dazu, Gott zu beschwichtigen' (Marx 2000:137). Sondern '... das Opfer [ist] Anlass des Kommens Gottes ..., um die Gastfreundschaft seines Volkes anzunehmen und es zu segnen' (Marx 2000:137). Übertragen auf Genesis 8:20f. hieße das: Das Opfer dient in diesem Text als Anlass für das Kommen Gottes, um die Gastfreundschaft Noahs anzunehmen und diesen sowie alle, die mit ihm zusammen die Flut überlebt haben, zu segnen. Diese Neudeutung des Opfers wird auch dadurch gestützt, dass sich hierin mit der Bedeutung des 'einladenden Duftes' gut einfügt. Nach der Errettung Noahs und seiner Familie vor der Flut wird Gott durch ein Brandopfer eingeladen, die bereits bestehende Kommunikation wieder aufzunehmen. Dies markiert zugleich den Neuanfang der nachsintflutlichen Menschheitsgeschichte. Und Gott lässt sich einladen. Ähnlich wie bei Atramhasis und Gilgameš kommt er. Seine Reaktion auf das Opfer entspricht genau der, die auch im Altargesetz Exodus 20 ankündigt wird: Er segnet die, die ihn eingeladen haben. ${ }^{41}$

35. Hierzu vergleiche insbesondere die Überblicksdarstellung von Willi-Plein (2011).

36.Dies geht vor allem auf die Thesen von Mauss (1990), Stansell (1999), Eberhart (2002), Kessler (2004) und Marx $(2000,2005)$ zurück. Exemplarisch hierzu Kessler (2004:402f.)

37.Marx (2000, 2005); Eberhart (2002).

38.So Marx (2000:136): 'Der ersten Art der Gastfreundschaft [bei der dem Gast das Mahl vom Gastgeber vorgesetzt wird, ohne dass dieser selbst am Mahl teilnimmt entspricht das Brandopfer, wo das ganze Tier Gott allein vorbehalten wird.' In die gleiche Richtung auch Willi-Plein (1993:84) zur מנחה: 'Aus der allgemeinen Huldigungsgabe ist dann eine - vielleicht auch nur metaphorisch gemeinte Gottesbewirtung geworden. Nie wird gesagt, dass Gott wirklich isst, aber es wird ihm aufgetragen wie einem feinen Herrn. Darum gehört zum Gesamtopfer de "liebliche Duft" oder "Beruhigungsduft" (reach nichoach), für den v.a. die Mincha sorgt. So stimmt sie Gott als den Audienz Gewährenden gnädig. Umso entsetzliche ist die prophetische Aussage (Am 5:21): "Ich mag eure Gottesdienste nich riechen." Denn normalerweise kann man einem Menschen, der in Schwierigkeiten ist, raten, Gott eine Mincha als Huldigungsgabe zu riechen zu geben (1 Sam 26:19).

39.So Marx (2000:136); er weist auch auf den dem Opfer innewohnenden Anthropomorphismus hin: 'Dass es [Israel] ihn [Gott] zu einem Opferschmaus einlädt, bezeugt, dass es inn für menschenähnlich hält. Denn nur jemand, der den Menschen ähnlich ist, kann an einem Mahl Gefallen haben. Wie es ihn empfängt, bezeugt demgegenüber den Rang, den es ihm beimisst.'

40.Marx (2000:137): 'Das Angebot der Gastfreundschaft setzt freundliche Beziehungen voraus, was nicht ausschließt, dass es auch zur Ehrung des Gastes dient, ja sogar zur Bekundung der Ehrfurcht und der Unterwerfung.'

41.Deutlicher noch wird Schenker (2003:145), der im Bandopfer Noahs - zumindest nach der Erzähllogik von Genesis 8:20f. - die Ursache für den Sinneswandel JHWHs sieht: 'Dieses Opfer begründet daher in der Erzählung die um 180 Grad veränderte Gesinnung JHWHs. (...) Es [sic] hält jedoch schwer, die kleine Erzählung von Gen 8:20-21 anders denn als logische Folge eines ursächlichen Zusammenhangs 8:20-21 anders denn als logische Folge eines ursächlichen Zusammenhangs
zu interpretieren. Die erzählende Abfolge kennt keine explizite oder implizite zu interpretieren.

\section{Fazit}

Was ergibt dieser religionsgeschichtliche Vergleich des Riechens des Opferdufts im Atramhasis-Mythos, im Gilgameš-Epos und in der biblischen Sintfluterzählung in Genesis 8:20f.?

1. Die drei Texte schildern göttliche Reaktionen auf das Opfer nach der Sintflut. Diese sehen im Einzelnen unterschiedlich aus:

- Die mesopotamischen Texte setzen einen doppelt ausgeführten Akzent: Götter und Menschen sind wechselseitig aufeinander angewiesen. Zum einen sind die Götter nach der opferlosen Sintflutzeit ausgehungert und scharen sich um den Opferduft. Zum anderen wird durch das verwendete Fliegenmotiv angedeutet, dass das Schicksal von Göttern und Menschen miteinander verknüpft ist.

- Demgegenüber beschreibt der biblische Text die positive Aufnahme des Opfers durch JHWH: Der Opferduft zieht ihn an. Von der Angewiesenheit JHWHs auf das Opfer ist hier nicht die Rede, und passend dazu wird auch das Fliegenmotiv nicht verwendet.

2. In allen drei Texten zieht diese unmittelbare göttliche Reaktion auf das Opfer Konsequenzen nach sich. Diese sehen wiederum im Einzelnen unterschiedlich aus:

- Die mesopotamischen Texte schildern zunächst den Ärger der Muttergottheit und den Streit innerhalb des Götterkollegiums. Aufgrund der sonst fehlenden Opfer beschließen die Götter gemeinsam, dass die Menschheit zukünftig nicht mehr komplett vernichtet werden soll. Selektive Dezimierungen sollen ausreichen.

- Imbiblischen Text folgt auf das Riechen des Opferdufts ein göttlicher Sinneswandel: Zwar hat JHWH seine Ansicht über den Menschen nicht verändert, aber trotzdem will er sie nicht mehr vernichten. Das Motiv dafür wird nicht explizit ungenannt. Vor dem Hintergrund der altorientalischen Texte und der neueren Opferdeutung in der alttestamentlichen Wissenschaft legt es sich nahe, dass die argumentative Lücke des Textes durch das Opfer gefüllt wird. Der göttliche Sinneswandel wird maßgeblich durch das Opfer herbeigeführt. Der Kontaktaufnahmeversuch des überlebenden Menschen in Gestalt des Opfers trifft auf eine positive Reaktion Gottes.

Offene Fragen bleiben in Hinblick auf das Opferverständnis. Hier wäre eine gründlichere Aufarbeitung der christlichen zumalderevangelischen-Sichtauf das Opfer wünschenswert. Wenn die vorliegenden Ausführungen zutreffend sind, dann wäre eine Höherbewertung des Opfers notwendig. Damit einhergeht eine Neubewertung der Kontaktaufnahme mit Gott sowie eine Revision der traditionell überlieferten Ablehnung des 'Anthropomorphismus' Gottes. ${ }^{42}$ Darüber hinaus müsste geprüft werden, ob nicht sinnliche Kommunikationswege stärker in die Theologie integriert

42.Anfragen aus anderer Perspektive gibt es an diese Vorstellung zum Beispiel durch Hartenstein (2008) sowie Wagner (2010). 
werden sollten. Ist es vielleicht sogar möglich, von einer 'Theologie des Riechens' im Alten Testament zu sprechen? ${ }^{43}$

\section{Konkurierende Interessen}

Die Verfasserin erklärt hiermit, daß sie keinerlei finanzielle oder persönliche Bindung hat, die sie zweckwidrig beim Schreiben dieses Artikels beeinflusste.

\section{Literaturliste}

Caduff, G.A., 1986, Antike Sintflutsagen, Vandenhoeck, Göttingen.

Eberhart, Chr., 2002, Studien zur Bedeutung der Opfer im Alten Testament. Die Signifikanz von Blut- und Verbrennungsriten im kultischen Rahmen, Neukirchener, Neukirchen-Vluyn. (WMANT 94).

Eberhart, Chr., 2011, 'Opfer und Kult in kulturanthropologischer Perspektive', Verkündigung und Forschung 1(56), 4-16.

García Martínez, F. \& Luttikhuizen, G.P. (eds.), 1998, Interpretations of the Flood, Brill, Leiden. (Themes in Biblical Narrative 1).

George, A.R., 2003, Babylonian Gilgamesh Epic, vol. 1, Oxford University Press, Oxford.

Gesenius, W., 1962, Hebräisches und aramäisches Handwörterbuch über das Alte Testament, bearbeitet von Frantz Buhl, Nachdruck der 17. Aufl. 1915, Springer, Berlin.

Gesenius, W., 2007, Hebräisches und aramäisches Handwörterbuch über das Alte Testament, bearbeitet von Herbert Donner, 18. Auflage, Lfg. 4, Springer, Berlin.

Gesenius, W., 2009, Hebräisches und aramäisches Handwörterbuch über das Alte Testament, bearbeitet von Herbert Donner, 18. Auflage, Lfg. 5, Springer, Berlin.

Hartenstein, F., 2008, Das Angesicht JHWHs:Studien zu seinem höfischen und kultischen Bedeutungshintergrund in den Psalmen und in Exodus 32-34, Mohr Tübingen. (FAT 55)

Hecker, K., 1994, 'Das akkadische Gilgamesch-Epos', TUAT III(4), 646-744. Gütersloher Verlagshaus, Gütersloh.

Jacob, B., 1934, Das Buch Genesis, Schocken, Berlin.

Jenni, E., 1981, Lehrbuch der hebräischen Sprache des Alten Testaments, 2. Aufl., Helbing \& Lichtenhahn, Basel.

Kessler, R., 2004, 'Die Theologie der Gabe bei Maleachi', in F.-L. Hossfeld u.a. (Hrsg.) Das Manna fällt auch heute noch. Beiträge zur Geschichte und Theologie des Alten, Ersten Testaments. Festschrift für Erich Zenger, pp. 392-407, Herder, Freiburg. (HBS 44).

Kilmer, A.D., 1987, 'The Symbolism of the Flies in the Mesopotamian Flood Myth and Some Further Implications', in F. Rochberg-Halton (ed.), Language, Literature, and History: Philological and Historical Studies Presented to Erica Reiner, pp. 175-180, American Oriental Society, New Haven, CT. (AOS 67).

Koch, K., 1986, Art. 'ניחוח nị̂âh', ThWAT V, 442-445.

Köhler, L., 1966, Theologie des Alten Testaments, 4. Auflage, Mohr, Tübingen.

Köhler, L. \& Baumgartner, W., 2004, Hebräisches und aramäisches Lexikon zum Alten Testament, 3. Aufl., Brill, Leiden.

Kronholm, T., 1993, Art. 'רוח rwh', ThWAT VII, 382-385.

Kügler, J. (Hrsg.), 2000, Die Macht der Nase. Zur religiösen Bedeutung des Duftes, Katholisches Bibelwerk, Stuttgart. (SBS 187).

43.Dazu vergleiche zum Beispiel Kügler (2000).
Lambert, W.G. \& Millard, A.R., 1969, Atrambasis: The Babylonian Story of the Flood, Clarendon, Oxford.

Lutherbibel: Die Bibel nach der Übersetzung Martin Luthers, revidierte Fassung 1984 Deutsche Bibelgesellschaft, Stuttgart.

Marx, A., 2000, 'Opferlogik im Alten Israel', in B. Janowski \& M. Welker (Hrsg.), Opfer. Theologische und kulturelle Kontexte, pp. 129-149, Suhrkamp, Frankfurt am Main.

Marx, A., 2005, Les systèmes sacrificiels de l'Ancien Testament. Formes et fonctions du culte sacrificiel à Yhwh, Brill, Leiden/Boston. (VT.S 105).

Maul, St., 2005, Das Gilgamesch-Epos, Beck, München.

Mauss, M., 1990, Die Gabe. Form und Funktion des Austauschs in archaischen Gesellschaften, Suhrkamp, Frankfurt am Main.

Porada, E., 1972, 'Problems of Iranian Iconography', in Memorial Volume of the Vth International Congress of Iranian Art \& Archaeology, pp. 163-182, Ministry of Culture and Arts, Teheran.

Rendtorff, R., 2004, Leviticus. 1. Teilband Leviticus 1,1-10,20, Neukirchener, Neukirchen-Vluyn. (BK.AT III/1).

Roth, M.T. (ed.), 1964-2010, s.v. 'incence offering', in I.J.Gelb (ed.), Assyrian Dictionary of the Oriental Institute of the University of Chicago (CAD), pp. 1956ff., Oriental Institute, Chicago, IL.

Sallaberger, W., 2008, Gilgamesch, Beck, München.

Schüle, A., 2009, Die Urgeschichte (Gen 1-11), Theologischer Verlag Zürich, Zürich (ZBK 1,1).

Schenker, A., 2003, 'Die Stiftungserzählung des Brandopfers. Wie versteht Gen 8:20-21 das Brandopfer?', in Studien zu Propheten und Religionsgeschichte, pp. 143-154, Katholisches Bibelwerk, Stuttgart. (SBAB 36).

Schmidt, B.B., 1995, 'Flood Narratives of Ancient Western Asia', in J. Sasson (ed.), Civilizations of the Ancient Near East III/IV, pp. 2337-2351, Hendrickson, Peabody, MA.

Seebass, H., 1996, Genesis, Bd. 1: Urgeschichte, Neukirchen-Vluyn, Neukirchener.

Stansell, G., 1999, 'The gift in ancient Israel', Semeia 87, 65-90.

Stolz, F., 1984, Art. 'נוח nua hu ruhen', THAT II (3. Auflage), 43-46.

Uehlinger, Chr., 1990, Weltreich und 'eine Rede'. Eine neue Deutung der sogenannten Turmbauerzählung (Gen 11,1-9), Universitätsverlag/Vandenhoeck, Fribourg/ Göttingen. (OBO 101).

Von Soden, W., 1965, s.v. 'Weihrauchopfer' in Akkadisches Handwörterbuch, Harrassowitz, Wiesbaden, Bd., S. 930.

Von Soden, W., 1994, 'Der altbabylonische Atramchasis-Mythos', TUAT III/4, 612-645, Gütersloher Verlagshaus, Gütersloh.

Wagner, A., 2010, Der Körper Gottes. Zur alttestamentlichen Vorstellung der Menschengestaltigkeit Gottes, Gütersloher Verlagshaus, Gütersloh.

Westermann, C., 1976, Genesis, 1. Teilband Genesis 1-11, 2. Aufl., Neukirchener, Neukirchen-Vluyn. (BK.AT I/1).

Wilcke, C., 1999, 'Weltuntergang als Anfang. Theologische, anthropologische, politischhistorische und ästhetische Ebenen der Interpretation der Sintflutgeschichte im babylonischen Atram-hasis-Epos', in A. Jones (Hrsg.), Weltende. Beiträge zur Kultur- und Religionswissenschaft, pp. 63-112, Harrassowitz, Wiesbaden.

Willi-Plein, I., 1993, Opfer und Kult im alttestamentlichen Israel. Textbefragungen und Zwischenergebnisse, Katholisches Bibelwerk, Stuttgart. (SBS 153).

Willi-Plein, I., 2011, 'Ein Blick auf die neuere Forschung zu Opfer und Kult im Alten Testament', Verkündigung und Forschung 1(56), 16-33.

Zwickel, W., 1994, Der Tempelkult in Kanaan und Israel. Studien zur Kultgeschichte Palästinas von der Mittelbronzezeit bis zum Untergang Judas, Mohr, Tübingen. (FAT 10). 